\title{
Neural network identification of a nonlinear model of a high-pressure sodium lamp
}

\author{
Anatolij D. Semenov ${ }^{1}$, Anton V. Volkov ${ }^{2, *}$, and Alena Yu. Shumkina ${ }^{2}$ \\ ${ }^{1}$ Department of Information Measuring Equipment and Metrology, Penza State University, Penza, \\ Russia \\ ${ }^{2}$ Department of Information Security and Service, National Research Mordovia State University, \\ Saransk, Russia
}

\begin{abstract}
The article is devoted to the identification of a high-pressure sodium lamp nonlinear model parameters based on neural network technologies. Identification was carried out using a dynamic neural network. The model had 17 parameters based on second order differential equations. As a result, out of 17 parameters, four were selected that accurately reflect the real picture of the model.
\end{abstract}

\section{Introduction}

Artificial neural networks (ANN) are a new and promising computing technology that allows in some cases very effectively solve computational problems in various areas of human activity. Applications of neural networks cover a wide range of areas of interest: pattern identification, data processing with noise, associative search, classification, optimization, forecasting, diagnostics, signal and image processing, control, management, data segmentation, information compression, complex modeling processes, artificial vision, and speech perception. The ability to model nonlinear processes, work with noisy data, and the ability to adapt allow us to use neural networks for solving a wide class of control problems [2].

Let us consider the construction and training of a two-layer dynamic neural network with linear activation functions that simulates the operation of a high-pressure sodium lamp. The change in parameters occurs due to changes in the parameters of the variables included in the equation (1) [1].

$$
\left\{\begin{array}{c}
\frac{d x_{1}}{d t}=\frac{1}{L}\left[U_{s}-\left(\frac{1}{x_{2} x_{3}}+R\right) x_{1}\right] \\
\frac{d x_{2}}{d t}=A_{l} U_{0}^{2} x_{2}^{2} \frac{\left(\frac{x_{1}}{U_{0} x_{2} x_{3}}\right)^{2}-1}{\mathbf{1}+\boldsymbol{k}_{1}\left(\frac{\left\lfloor x_{1}\right\rfloor}{U_{0} x_{2} x_{3}}-\mathbf{1}\right)} \\
\frac{d x_{3}}{d t}=\left[k_{2}+k_{3}\left(\frac{\left\lfloor x_{1}\right\rfloor}{U_{0} x_{2} x_{3}}\right)^{k_{4}}\right]\left[1+\boldsymbol{k}_{\mathbf{1}}\left(\frac{\left.\mid x_{1}\right\rfloor}{U_{0} x_{2} x_{3}}-\mathbf{1}\right)-x_{3}\right]
\end{array}\right.
$$

\footnotetext{
* Corresponding author: elsoldador@rambler.ru
} 
where $x_{1}$ is lamp current; $x_{2}$ is the reduced conductivity of the lamp taking into account the average value of the electron concentration; $x_{3}$ is a dimensionless quantity that takes into account electron mobility; $L, R$ are the inductance and active resistance of the limiting choke respectively; $U_{s}, U_{0}$ are the supply voltage and the rated voltage on the lamp respectively; $A_{l}$ is a coefficient determined by the design of the lamp; $\boldsymbol{k}_{\mathbf{1}}-k_{4}$ are electrical coefficients determined for a specific type of lamp.

Figure 1 shows the results of modeling a high-pressure sodium lamp using a two-layer dynamic neural network with a linear activation function.
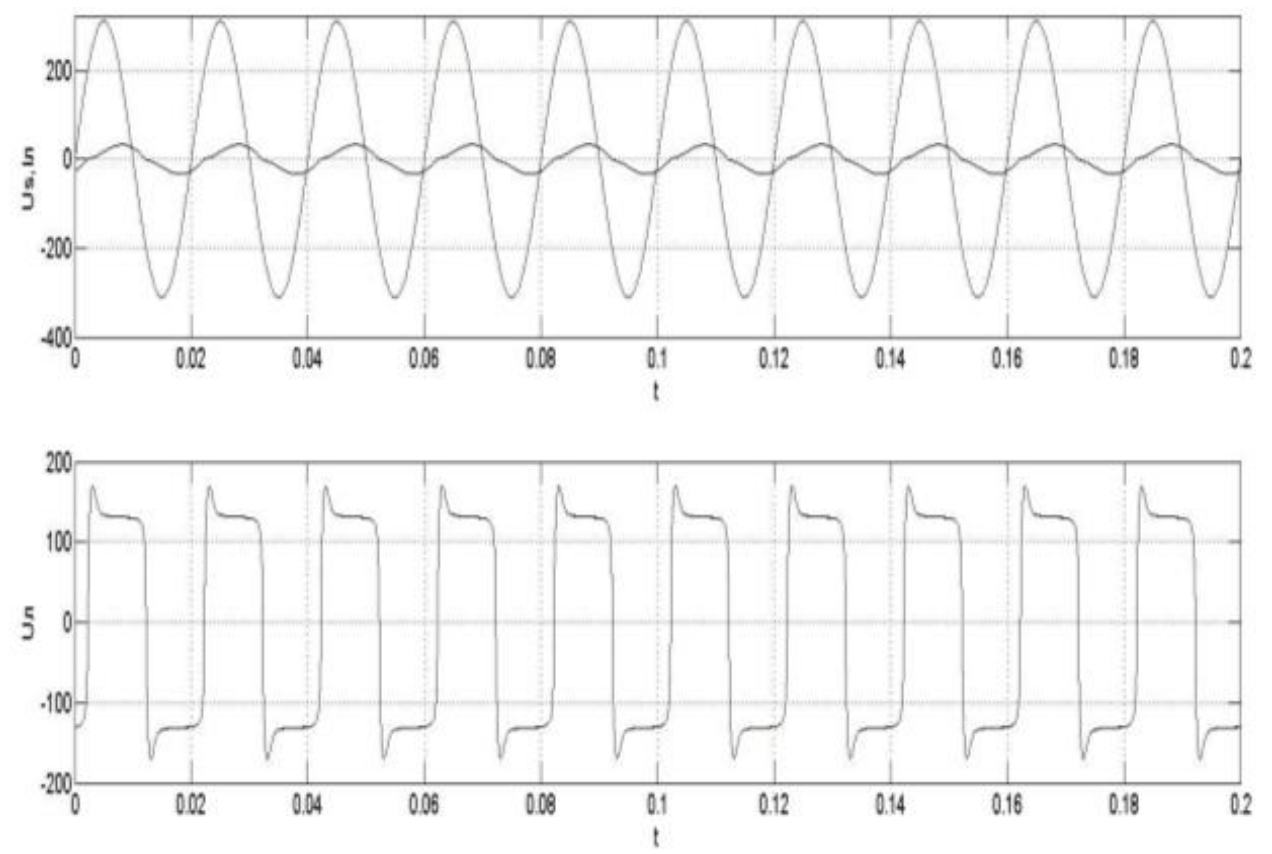

Fig. 1. Simulation results of a high-pressure sodium lamp.

The parameters obtained from equation (1) were used as a training sample. The training was carried out using the error back propagation algorithm using the Levenberg -Marquardt method $[3,4]$.

The result is a neural network with the structure shown in figures $2-3$. The first layer are used two neurons and the second layer is used one. The training error of such a network is almost zero [4].

The neural model based on a dynamic two-layer neural network is characterized by 17 parameters representing synaptic (weight) coefficients of the network. The simulation error is almost zero. 


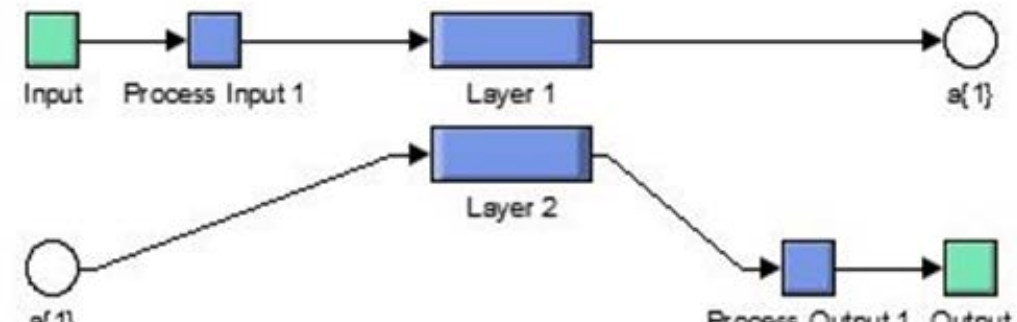

$\{1\}$

Process Output 1 Output

$\mathrm{a}$
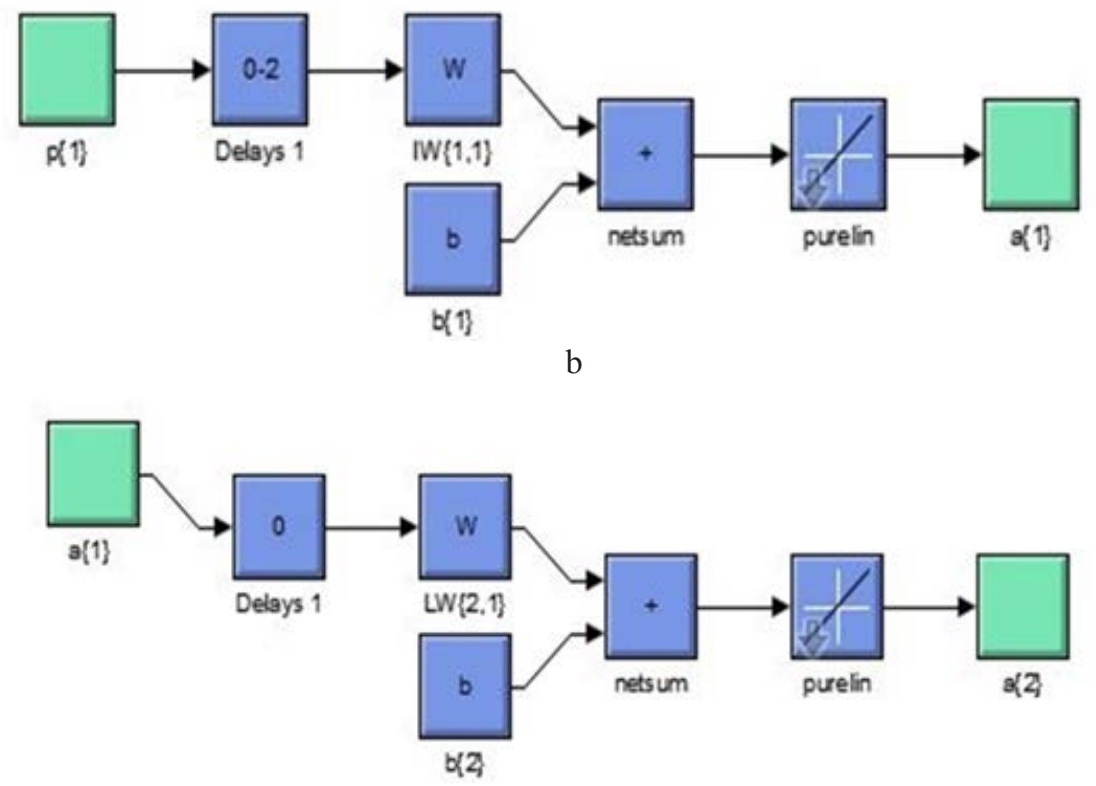

c

Fig. 2. Neural network structure, where $a$ - a two - layer neural network; $b$ - structure of the first layer; $c$-structure of the second layer 


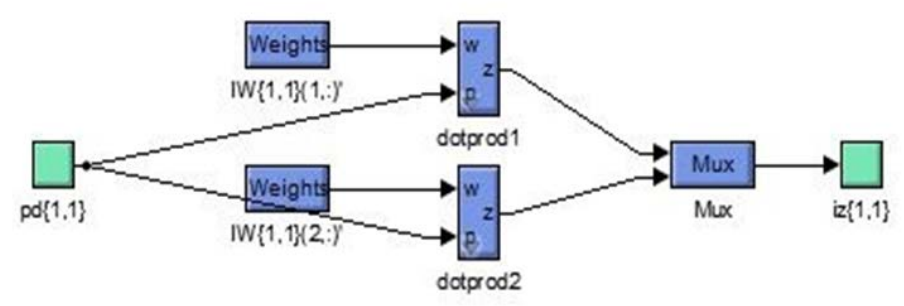

a

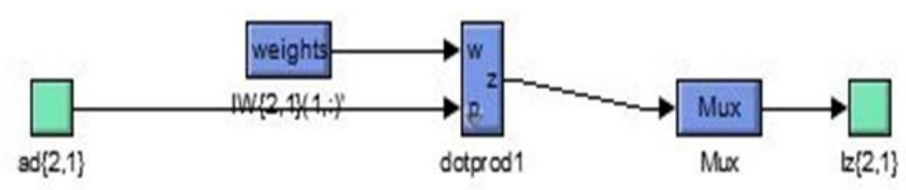

b

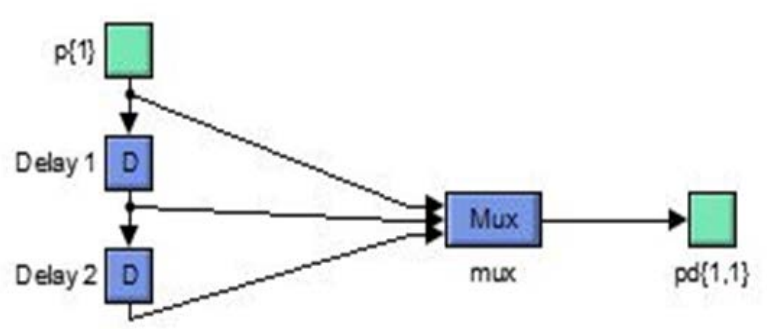

C

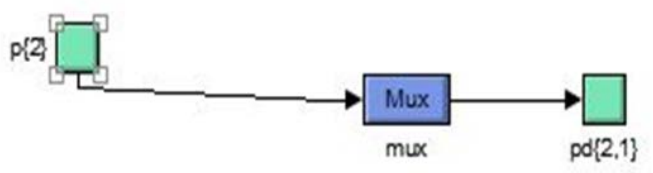

d

Fig. 3. Neural network structure, where $a$-input coefficients of the dendrite of the first layer; $b-$ input coefficients of the dendrite of the second layer; $c$ - activation function for the first layer; $d-$ activation function for the second layer.

The author proposed to expand the number of measured parameters of the lamp, adding it in addition to the voltage drop on the lamp with the current consumed by it to improve the accuracy of monitoring high-pressure sodium lamps. Expanding the number of measured parameters allowed us to develop a number of refined mathematical models of the lamp, which differ in various degrees of detail of the physical processes occurring in it. A neural network model is synthesized on base a two-layer dynamic neural network. The model error is almost zero. The parametric model identification is complex and requires the use of neural network technologies. The number of identified parameters is 17 . 
Experimental verification of the obtained models showed that the use of a neural network provides minimal modeling error.

\section{References}

1. A.V. Volkov, Sc. and Tech. Volga reg. Bull., 8, 65-68, (2018).

2. A.I. Galushkin, The theory of neural networks. B. 1 (IPRZhR, Moscow 2000).

3. V.V. Volkov, I.I. Conovalova, A.D. Semenov, Continuous process management in technical systems, URL: https://e.lanbook.com/book/62610.

4. V.P. Diakonov, MATLAB 6.5 SP1/7/7 SP1/7 SP2 + Simulink 5/6. Artificial intelligence and bioinformatics tools (Solon-Press, Moscow, 2009). 